\title{
A comparative study of uniaxial pressure effects in intraband AlGaAs/GaAs and interband InAs/AISb/GaSb resonant tunneling diodes
}

\author{
K. Mutamba, ${ }^{\text {a) }}$ A. Sigurdardóttir, A. Vogt, and H. L. Hartnagel \\ Institut für Hochfrequenztechnik, Technische Universität Darmstadt, D-64283 Darmstadt, Germany \\ E. H. Li \\ Department of Electrical and Electronic Engineering, The University of Hong Kong, Hong Kong
}

(Received 19 November 1997; accepted for publication 3 February 1998)

\begin{abstract}
We report on the effects of uniaxial pressure on (001)-oriented $\mathrm{AlGaAs} / \mathrm{GaAs}$ and InAs/AlSb/GaSb double barrier resonant tunneling diodes (RTDs). The current-voltage characteristics of the AlGaAs/GaAs RTDs shift asymmetrically due to stress-induced piezoelectric fields in the barriers and well structures. Although all the materials involved are piezoelectric, the interband InAs/AlSb/ $\mathrm{GaSb}$ resonant tunneling device surprisingly shows, in contrast to the $\mathrm{AlGaAs} / \mathrm{GaAs}$ one, a symmetrical behavior for the same orientation [110] of the applied pressure. We explain the observed differences considering the different tunneling paths involved in the conduction mechanism of the two heterostructure device types as well as their pressure dependencies. (C) 1998 American Institute of Physics. [S0003-6951(98)03013-7]
\end{abstract}

Pressure or stress plays a very important role in the investigation of the transport properties in semiconductor materials and offers a possibility of designing heterostructure devices with tailorable performances. A variety of pressure effects has been obviously used in diverse integrated semiconductor sensors with an improved degree of miniaturization. ${ }^{1-3}$ The well-known effects are: the energyband-gap variation with hydrostatic pressure, the stressinduced piezoelectric field in III-V compound semiconductors, and the change of the band-edge curvature and the band-edge splitting with uniaxial pressure. ${ }^{4}$ Therefore, the band structure and the line-up of the band discontinuities in heterostructure devices result in a defined pressure dependence of the transport properties. The possibility of epitaxial growth of strained layers has opened a way to control this dependence by controlling the amount of the built-in strain. Modified hole effective masses could be obtained in strained InGaAs layers on GaAs resulting, for example, in highperformance modulation-doped field-effect transistors and quantum well lasers. ${ }^{5,6}$ The strain-generated piezoelectric fields in InGaAs/GaAs quantum well structures and the resulting fields screening are also of great interest for electrooptical devices. $^{7}$

In the former pressure experiments on resonant tunneling diodes (RTDs), pressure has proved to be a means of introducing participation of different band edges of the barrier and well materials to the transport mechanisms. ${ }^{8}$ The observed relative shifts of the negative differential resistance region are symmetric for hydrostatic and [100]-oriented uniaxial pressures. ${ }^{9}$ A special dependence has been observed with [110]-oriented uniaxial pressure on $\mathrm{AlGaAs} / \mathrm{GaAs}$ RTDs. ${ }^{10,11}$ The pressure-induced shift of the current-voltage characteristics is asymmetric and this can be attributed to the presence of piezoelectric fields in the barriers and well of the RTD. All III-V compounds are piezoelectric due to the polar

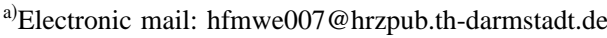

character of the bonds between different atoms. ${ }^{12}$ The same behavior can then be, in a first approximation, expected with RTDs based on other III-V compounds for the same wafer orientation and pressure direction. ${ }^{13}$ In this letter, we report on the special symmetric shift of the resonance region in interband InAs/AlSb/GaSb RTDs. We explain this effect and the difference to the AlGaAs/GaAs RTDs with the nature of the tunneling channel involved in the interband RTD. In the interband device, the resonant electrons tunneling takes place through intermediate states in the well formed in the valence band of GaSb. Note that the possibility of obtaining a high peak-to-valley ratio, due to very low reachable valley currents, has motivated the growing interest on interband resonant tunneling diodes.

The used resonant tunneling diodes were grown by molecular beam epitaxy (MBE) on (001)-oriented GaAs substrates. The corresponding band diagrams are shown in the Fig. 1. The double barrier structure of the AlGaAs/GaAs RTD consists of a $4 \mathrm{~nm}$ undoped GaAs well between two 3 nm undoped $\mathrm{Al}_{0.6} \mathrm{Ga}_{0.4} \mathrm{As}$ barriers. On both sides of the double barrier, undoped $15 \mathrm{~nm}$ GaAs spacer layers and 50 nm $n$-GaAs supply layers $\left(10^{17} \mathrm{~cm}^{-3}\right)$ were grown. The $n^{+}$-GaAs top and bottom contact layers $\left(10^{18} \mathrm{~cm}^{-3}\right)$ are, respectively, $700 \mathrm{~nm}$ and $1 \mu \mathrm{m}$ thick. Ni/AuGe/Ni Ohmic

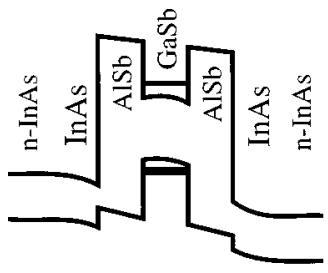

(a)

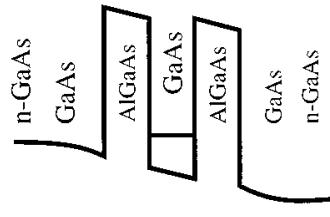

(b)
FIG. 1. Respective RTD band diagrams under applied voltage: (a) InAs/ $\mathrm{AlSb} / \mathrm{GaSb}$ interband RTD, conduction- and valence-band profiles are involved. The electron tunneling occurs via an intermediate hole state in the $\mathrm{GaSb}$ well. (b) AlGaAs/GaAs intraband RTD, only conduction-band and electron energy states are involved. 


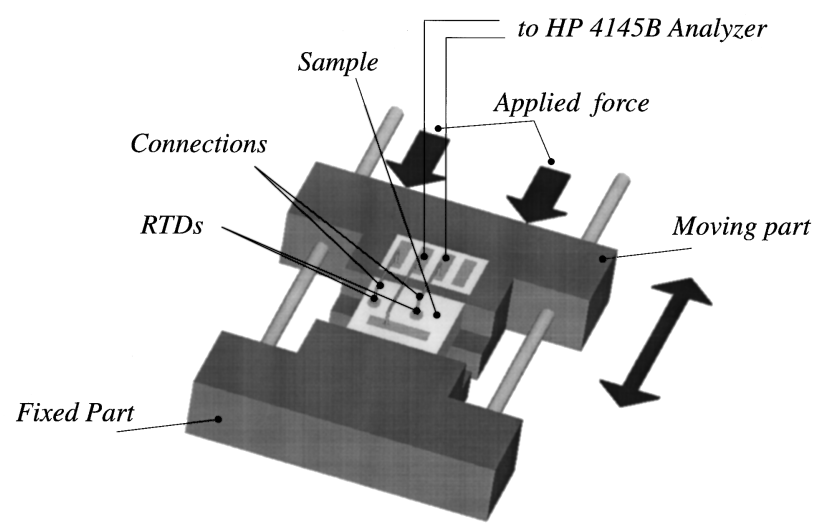

FIG. 2. Schematic of the pressure-dependent measurement setup. The apparatus stands vertically and the moving part is linked to a lever system.

contacts have been used. The parameters of the MBE-grown interband $\mathrm{InAs} / \mathrm{AlSb} / \mathrm{GaSb}$ device were an undoped $5 \mathrm{~nm}$ GaSb well, $9 \mathrm{~nm}$ undoped AlSb barriers, $10 \mathrm{~nm}$ undoped InAs spacer layers, $50 \mathrm{~nm}$ InAs supply layers $\left(10^{17} \mathrm{~cm}^{-3}\right)$, and $500 \mathrm{~nm}\left(10^{18} \mathrm{~cm}^{-3}\right)$ for the top and the bottom contacts, respectively. A buffer structure consisting of $2.5 \mathrm{~nm}$ AlAs and $500 \mathrm{~nm} \mathrm{AlSb}$ was grown prior to the double barrier structure in order to reduce the amount of dislocations generated by the lattice mismatch between InAs and GaAs. Nonalloyed $\mathrm{Cr} / \mathrm{Au} \mathrm{Ohmic}$ contacts have been used.

Measurements have been performed on $100 \mu \mathrm{m}$ diam devices. A native acceptor concentration of $10^{16} \mathrm{~cm}^{-3}$ has been measured for the unintentionally doped $\mathrm{GaSb}$ well. Compressive uniaxial pressures were applied in the [110] direction using a measurement setup with a lever system and the possibility of applying forces of defined magnitudes on the sample edges (Fig. 2). The measured pressure-dependent current-voltage characteristics of the two device types at room temperature are presented in Figs. 3(a) and 3(b). Pressures of up to 205 bar have been used. The weakness of the negative resonance of the InAs/AlSb/GaSb device at room temperature is due to an asymmetry in the growth of the structure. The $77 \mathrm{~K}$ characteristics in the inset show a pronounced resonance occurring in the same voltage region.

The description of the asymmetric shift in $\mathrm{AlGaAs} / \mathrm{GaAs}$ RTDs is based on the assumption that there are no free carriers in the undoped double barrier structure. This does not allow the stress-induced polarization charges appearing at the layers interfaces to be totally compensated. The resulting electrical fields in the well shifts the resonance position depending on the biasing direction. In the InAs/AlSb/GaSb RTD, the high hole concentration obtainable without intentionally doping $\mathrm{GaSb}$ leads to a compensation of polarization charges appearing at the interfaces to the barriers. ${ }^{14}$ As far as the trend in the change of the $I-V$ characteristics are concerned, the difference in the peak current densities of the two used devices do not have an impact on the experiment. In a coherent model, the peak current density is mainly determined by the thickness and height of the barriers. It is dependent, to some extent, of the carrier effective masses in the different materials. The observed behavior is related, as explained below, to the position of the quantized energy levels as well as the band edge in the well material. These depend (a)

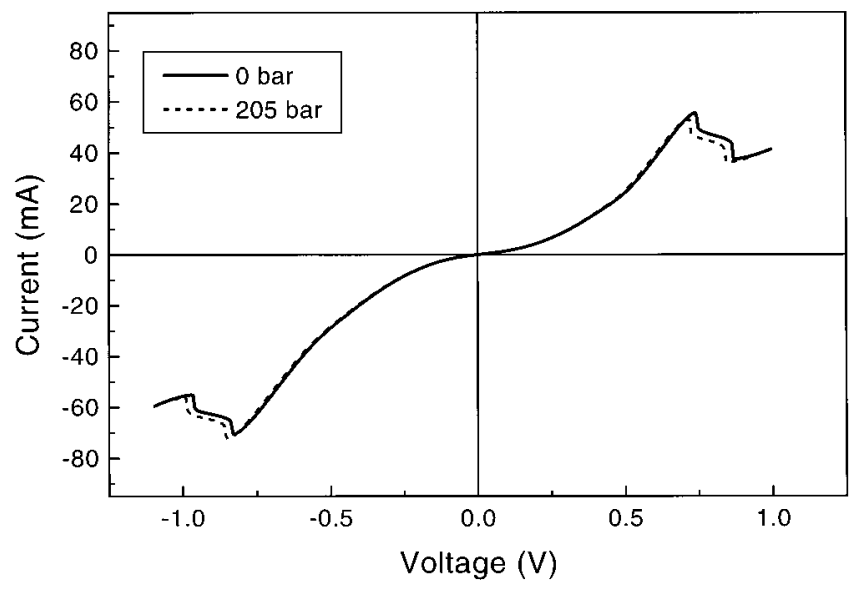

(b)

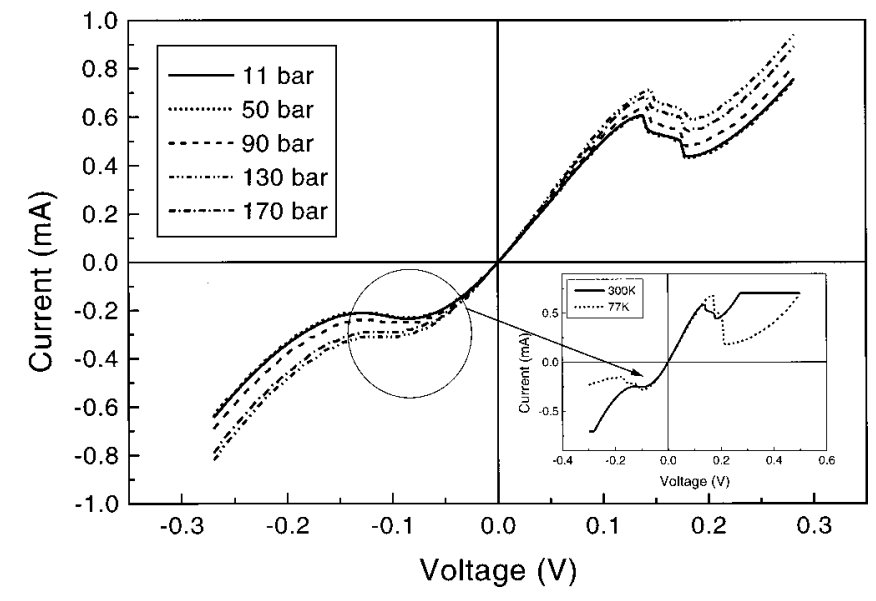

FIG. 3. Uniaxial pressure-dependent RTD current-voltage characteristics: (a) AlGaAs/GaAs RTD: the uniaxial pressure induced shift is assymmetric. (b) InAs/AlSb/GaSb RTD: the shift is symmetric. The inset shows a pronounced resonance occurring in the same negative voltage region as for the measurements at room temperature.

on the well width, the barrier height, and the material band structure. However, in a realistic model, influences such as scattering due to the quality of the different interfaces as well as their possible pressure dependence should be taken into account.

A general description of the electrical conductivity in III-V compounds under applied uniaxial pressure can be described by the relation between electric-field $E_{i}$ and current $i_{j}:{ }^{15}$

$$
d E_{i}=\rho_{i j} d i_{j}+d_{i k l} \sigma_{k l}+\pi_{i j k l} \sigma_{k l}+f l,
$$

where $\rho_{i j}$ is the resistivity, $\sigma_{k l}$ is the uniaxial pressure or stress, $d_{i k l}=\partial E_{i} / \partial \sigma_{k l}$ are the piezoelectric coefficients, and $\pi_{i j k l}=\partial^{2} E_{i} / \partial i_{j} \partial_{k l}$ are the piezoresistance coefficients. The remaining terms of Eq. (1), which include the change in resistivity with current as well as the change in piezoelectric coefficients with stress can be neglected.

The piezoelectric effect will be absent in doped bulk materials for static measurements. Piezoresistance is static and has a magnitude, which depends on both the doping type and level. It is worth noting that speaking about piezoresistance here implies the primary effects, which are the band

splitting and the carrier effective-mass change. 
For the AlGaAs/GaAs RTDs, the major change in the I-V characteristics with pressure occurs in the resonance region and there is no notable change in the positive resistance regions and around the zero-voltage point. This proves that piezoresistance, which can be assumed to occur in the $n$-doped GaAs supply layers of the RTD can be neglected. This is also supported by the very weak piezoresistance coefficients of $n$-doped GaAs. ${ }^{16}$ However, the effect of piezoresistance can be clearly seen in the $I-V$ characteristics of InAs/AlSb/GaSb interband RTDs and a qualitative description is possible. A change of about $7 \% / \mathrm{kbar}$ in the current could be deduced from the measurements in the positive conductance region before the resonance. In this case, the uniaxial stress can change the Fermi-level position in the InAs contact layers, the hole effective mass, and the valenceband edge in the GaSb well. We suggest that the two latter effects are dominant for the pressure-induced changes. In fact, a calculated heavy- and light-holes energy-band splitting of about $6 \mathrm{meV} / \mathrm{kbar}$ can be obtained with respect to the wafer orientation and the uniaxial stress direction. ${ }^{17}$ The contribution from the hole effective-mass change can be explained as follows: with respect to light holes and the related energy states, the applied compressive pressure increases the well depth and shifts the voltage at which the band-gap blocking occurs at higher values. ${ }^{4}$

A comparative study of uniaxial pressure effects in intraband AlGaAs/GaAs and interband InAs/Alsb/GaSb has been carried out. The special dependence in the interband case can be explained by including piezoresistance in the conduction mechanisms. However, some complementary calculations, such as the coupling between the light- and heavy-hole bands will be necessary for a more quantitative description, which could allow one to tailor device performances.
This work was supported by the DAAD (Reference No. 423-PPP-hk)-RGC joint project between the DAAD and the Research Grant Council of Hong Kong.

${ }^{1}$ K. Fricke and H. L. Hartnagel, Electron. Lett. 26, 693 (1990).

${ }^{2}$ A. Dehé, K. Fricke, K. Mutamba, and H. L. Hartnagel, J. Micromech. Microeng. 5, 139 (1995).

3 J. L. Robert, V. Moser, and S. Contreras, Proc. Transducers '91, pp. $294-$ 299, Yokohama, Japan (1991).

${ }^{4}$ S. C. Jain, M. Wilander, and H. Maes, Semicond. Sci. Technol. 11, 641 (1996).

${ }^{5}$ T. Henderson, M. I. Aksun, C. K. Peng, H. Morkoc, P. C. Chao, P. M. Smith, K.-H. G. Duh, and L. F. Fester, IEEE Trans. Electron Devices 7, 649 (1986).

${ }^{6}$ P. J. Thjis, L. F. Tiemejer, J. J. M. Binsma, and T. van Dongen, IEEE J. Quantum Electron. 30, 477 (1994).

${ }^{7}$ P. J. Rodriguez-Girones and G. J. Rees, IEEE Photonics Technol. Lett. 7, 71 (1995).

${ }^{8}$ D. G. Austing, P. C. Klipstein, J. S. Roberts, and G. Hill, Semicond. Sci. Technol. 10, 616 (1995).

${ }^{9}$ D. G. Austing, P. C. Klipstein, A. W. Higgs, G. W. Smith, J. S. Roberts, and G. Hill, NATO ASI Ser. B 277, 157 (1991).

${ }^{10}$ K. Mutamba, A. Vogt, A. Sigurdardottir, J. Miao, A. Dehé, I. Aller, and H. L. Hartnagel, "Uniaxial stress dependence of AlGaAs/GaAs RTD characteristics for sensor applications," Proceedings of the Micromechanics Europe Conference MME'96, Barcelona, Spain (1996).

${ }^{11}$ K. Fobelets, R. Vounckx, and G. Borghs, J. Micromech. Microeng. 4, 123 (1994).

${ }^{12}$ K. Hjort, Ph.D. thesis, University of Uppsala, Sweden (1993).

${ }^{13}$ A. D. Bykhovski, B. L. Gelmont, and M. Shur, J. Appl. Phys. 81, 6332 (1997).

${ }^{14}$ P. S. Dutta, H. L. Bhat, and Vikram Kumar, J. Appl. Phys. 81, 5821 (1997).

${ }^{15}$ L. E. Hollander, G. L. Vick, and T. J. Diesel, Rev. Sci. Instrum. 31, 323 (1960).

${ }^{16}$ Landolt and Börnstein, Numerical Data and Functional Relationships in Science and Technology, New Series, Group III: Crystal and Solid State Physics, Vol. 17 Semiconductors (Springer, Berlin, 1984).

${ }^{17}$ J. C. Hensel and G. Feher, Phys. Rev. 129, 1041 (1963). 